\title{
Implante de Schocket modificado em glaucomas refratários: resultados a longo prazo
}

\author{
Modified Schocket implant for refractory glaucoma:long-term results
}

\author{
João Antonio Prata Jr ${ }^{(1)}$ \\ Antonio Eduardo Pereira ${ }^{(2)}$ \\ Carlos Akira Omi ${ }^{(3)}$
}

\begin{tabular}{|l|}
\hline \multicolumn{1}{|c|}{ RESUMO } \\
\hline Objetivo: Analisar os resultados a longo prazo obtidos com o implante \\
deSchocket modificado. \\
Métodos: Os prontuários de 45 pacientes (45 cirurgias) submetidas \\
ao implante de Schocket modificado com pelo menos um ano de \\
acompanhamento foram analisados. Estudou-se as taxas de sucesso \\
(sucesso=Po<22 mmHg), tempo de sobrevida, diagnóstico, acompa- \\
nhamento pós-operatório, Po inicial e final, redução percentual pós- \\
operatória da pressão intra-ocular (Po) e complicações. \\
Resultados: Após um acompanhamento médio de $31,9 \pm 19,9$ meses 28 \\
casos (62,2\%) foram considerados sucesso. O tempo médio de sobrevida \\
foi de 38,9 meses com probabilidade de sucesso de 0,74 por volta dos 30 \\
meses. A Po inicial foi de $37,2 \pm 11,3$ e a final de $16,3 \pm 9,3$ mmHg, com \\
reduçãode $49,6 \pm 32,6 \%$. A análise das taxas desucesso e sobrevida entre \\
os diferentes grupos diagnósticos (glaucoma e afacia, neovascular, \\
congênito e pós-ceratoplastia) não revelou diferença estatisticamente \\
significante. \\
Conclusão: Os resultados sugerem que o implante de Schocket \\
modificadopropicia um controle satisfatório de glaucomas refratários a \\
longoprazo.
\end{tabular}

Palavras-chave: Glaucoma; Implantes oculares.

\section{INTRODUÇÃOO}

A literatura dos últimos anos tem salientado a significativa eficácia dos implantes para glaucoma no controle da pressão intra-ocular (Po) de glaucomas rebeldes às formas convencionais de tratamento cirúrgico ${ }^{1-3}$. Esses dispositivos foram incorporados à rotina cirúrgica do especialista em glaucoma e têm permitido a solução de casos que anteriormente evoluiam de forma desfavorável.

Atualmente são disponíveis diversos tipos de implantes para glaucoma, sendo que com praticamente todos os modelos, estudos referem resultados satisfatórios ${ }^{1-6}$. Basicamente, as principais diferenças entres os vários tipos de implantes residem na forma e dimensões da região de drenagem do dispositivo e na presença ou não de restrictor de fluxo ${ }^{7,8}$.

$\mathrm{O}$ custo da maioria dos implantes para glaucoma disponíveis no mercado internacional é muitas vezes incompatível com os recursos financeiros existentes, acarretando a adoção de técnicas mais adequadas a realidade da população ${ }^{7}$. Por essa dentre outras razões, o implante de Schocket modificado vem sendo utilizado há alguns anos e diversos estudos ressaltam a sua eficácia ${ }^{4,6,9,10}$. É facilmente acessível, tendo em 
vista que é confeccionado pelo próprio cirurgião momentos antes do procedimento, utilizando materiais facilmente disponíveis e a baixo custo ${ }^{4,6,9,10}$.

Em publicação anterior, um dos autores Omi et al. ${ }^{10}$ apresentou os resultados observados com o implante de Schocket modificado em glaucomas refratários após um acompanhamento pós-operatório médio de 10,3 meses. O objetivo do presente estudo é apresentar os resultados a longo prazo obtidos com o implante de Schocket modificado.

\section{MATERIAL E MÉTODOS}

Foram revisados todos os prontuários de pacientes submetidos à cirurgia com o implante de Schocket modificado para o controle da Po no Setor de Glaucoma do Departamento de Oftalmologia da Universidade Federal de São Paulo Escola Paulista de Medicina. Foram incluídos somente os casos em que o tempo de acompanhamento pós-operatório foi igual ou superior a um ano. Casos com complicações intraoperatórias foram excluídos e quando de cirurgia bilateral, somente o primeiro olho operado foi incluído.

Das informações disponíveis nos prontuários, foram anotados os dados demográficos, diagnóstico, tipo de cirurgias prévias, cirurgias concomitantes, Po pré e pósoperatórias, presença de complicações e uso de medicações tópicas ou sistêmicas. Considerou-se como exame préoperatório, o obtido no dia da indicação cirúrgica.

A técnica cirúrgica empregada foi a mesma em todos os casos e basicamente consistiu de incisão conjuntival de base límbica a $8 \mathrm{~mm}$ do limbo em um quadrante do globo ocular (geralmente o temporal superior) onde procedeu-se a divulsão do espaço subconjuntival, identificação e isolamento dos músculos extra-oculares adjacentes. O implante de Schocket modificado foi fixado à esclera a pelo menos $10 \mathrm{~mm}$ do limbo sob dois músculos retos, com o uso de dois pontos de fio inabsorvível. A porção anterior do tubo foi cortada a fim de que o tubo dentro da câmara anterior permanecesse a uma distância eqüidistante entre a raiz da íris e o bordo pupilar. A introdução do tubo na câmara anterior foi procedida através da confecção de um túnel límbico com o auxílio de agulha calibre 23. O trajeto do tubo foi recoberto com um enxerto de esclera conservada. A síntese conjuntival foi realizada com o uso de ponto contínuo com fio absorvível. A fim de prevenir a ocorrência de hipotensão pós-operatória precoce, em todos os casos o tubo foi totalmente ocluído com fio absorvível posicionado próximo da porção posterior do implante. A preparação do implante de Schocket foi realizada conforme anteriormente publicado ${ }^{10}$.

Pós-operatoriamente, todos os casos receberam colírios de corticosteróide, antibiótico e cicloplégico e a medicação antiglaucomatosa foi mantida até a absorção da ligadura do tubo (2-3 semanas).

Além dos dados demográficos foram comparados no préoperatório a freqüência de tipos diagnósticos, tipo de cirurgias prévias e nível da Po. No pós-operatório foram comparados os valores da Po final (Po na última visita), bem como a redução percentual da Po pós-operatória em relação à inicial. Considerou-se como sucesso cirúrgico a Po final abaixo de $22 \mathrm{mmHg}$, sem a ocorrência de complicações devastadoras (phythisis bulbar, descolamento de retina). O sucesso cirúrgico foi avaliado através de tabela de vida pelo teste de Kaplan-Meier. Foram analisadas também as freqüências de sucesso e de complicações pós-operatórias. As variáveis acima foram comparadas entre grupos de diagnóstico compostos por mais de 5 casos.

\section{RESULTADOS}

Foram analisadas 45 cirurgias (45 pacientes). A tabela I apresenta os dados demográficos e distribuição quanto ao diagnóstico. O implante de Schocket modificado foi realizado como primeiro procedimento cirúrgico antiglaucomatoso em 17 casos (37,8\%). Em 19 pacientes havia sido realizada pelo menos uma trabeculectomia $(42,2 \%)$. Cinco $(11,1 \%)$ haviam sido submetidos a uma cirurgia prévia com tubo de drenagem, três $(6,7 \%)$ à trabeculectomia e posterior tubo de drenagem e 1 à goniotomia (Tab. I). Cirurgias concomitantes foram realizadas em 19 casos $(42,2 \%)$. Nove $(20 \%)$ foram tratados com pan-crioablação de retina periférica (glaucomas neovasculares) e $10(22,2 \%)$ com vitrectomia via pars-plana.

O tempo médio de acompanhamento pós-operatório foi de $31,9 \pm 19,9$ meses (12,1-103 meses) (Tab. II). 9 casos foram observados por um período entre 12 a 17 meses, 9 entre 18 e 23 meses, 12 entre 24 e 35 meses e 15 por um tempo igual ou superior a 36 meses ( 9 acima de 48 meses).

$\mathrm{Na}$ última visita, 28 casos $(62,2 \%)$ foram considerados su-

\begin{tabular}{|lll|}
\hline & Tabela I - Dados pré-operatórios & \\
$\mathrm{n}$ & & 45 \\
Idade média & & $43,9 \pm 23,3$ \\
Raça & & \\
& Branca & $28(62,2 \%)$ \\
Sexo & Não-branca & $17(27,8 \%)$ \\
& & \\
& Feminino & $28(62,2 \%)$ \\
Diagnóstico & Masculino & $17(27,8 \%)$ \\
& & \\
& afácico & 16 \\
& neovascular & 10 \\
& congênito & 7 \\
& pós-ceratoplastia & 10 \\
Tipo de cirurgias prévias & 1 \\
& pós-trauma & 1 \\
& pós-uveíte & \\
& nenhum & $17(37,8 \%)$ \\
& trabeculectomia & $19(42,2 \%)$ \\
& trabeculecomia e tubo & $03(06,7 \%)$ \\
& tubo de drenagem & $05(11,1 \%)$ \\
& goniotomia & $01(02,2 \%)$ \\
\hline
\end{tabular}




\begin{tabular}{|c|c|}
\hline \multicolumn{2}{|c|}{$\begin{array}{l}\text { Tabela II - Acompanhamento, pressão intra-ocular, sucesso e } \\
\text { tempo de sobrevida }\end{array}$} \\
\hline $\mathrm{n}$ & 45 \\
\hline Acompanhamento & $31,9 \pm 19,9$ \\
\hline \multicolumn{2}{|l|}{ pós-operatório (meses) } \\
\hline $12-18$ meses & $09(20 \%)$ \\
\hline 18-24 meses & $09(20 \%)$ \\
\hline 24-36 meses & $12(26,7 \%)$ \\
\hline acima de 36 & $15(33,3 \%)$ \\
\hline Po pré $(\mathrm{mmHg})$ & $37,2 \pm 11,3$ \\
\hline Po final $(\mathrm{mmHg})$ & $16,3 \pm 9,3$ \\
\hline redução \% Po & $49,6 \pm 32,6 \%$ \\
\hline Sucesso & $28(62,2 \%)$ \\
\hline sem medicação & $11(24,5 \%)$ \\
\hline com medicação & $17(37,7 \%)$ \\
\hline Insucesso & $17(37,7 \%)$ \\
\hline Tempo de sobrevida (meses) & $38,9(35,1-42,8 \mathrm{IC} 95 \%)$ \\
\hline
\end{tabular}

cesso, 11 sem o uso de antiglaucomatosos e 17 com medicação hipotensora. Dezessete cirurgias $(37,7 \%)$ foram consideradas insucesso, 12 devido a Po acima de $22 \mathrm{mmHg}, 3$ devido a ocorrência de phytisis bulbar (todos glaucoma neovascular) e dois por descolamento de retina (pós-ceratoplastia) (Tab. II). Os valores médios da Po inicial, final e da redução percentual média da Po em relação à inicial encontram-se na tabela II. O tempo médio de sobrevida foi de 38,9 meses com uma probabilidade de sucesso de 0,74 por volta dos 30 meses de pós-operatório e de 0,5 aos 46,4 meses (Graf. 1).

A tabela III apresenta as freqüências de casos considerados sucesso por grupo diagnóstico, tempo de acompanhamento pós-operatório, bem como, a Po inicial, final e redução percentual média em relação à Po inicial e o tempo de sobrevida da cirurgia. Não foram observadas diferenças estatísticas quanto à duração do período de acompanhamento pós-operatório entre os grupos diagnósticos, como também para as taxas de sucesso. A análise da probabilidade de sobrevida pelo teste de Kaplan-Meier não demonstrou diferenças estatísticas entre os grupos diagnósticos $(\mathrm{p}=0,8-\log$ rank) (Graf. 2).

A comparação da Po inicial entre os diferentes tipos de diagnóstico pela análise de variância mostrou significância
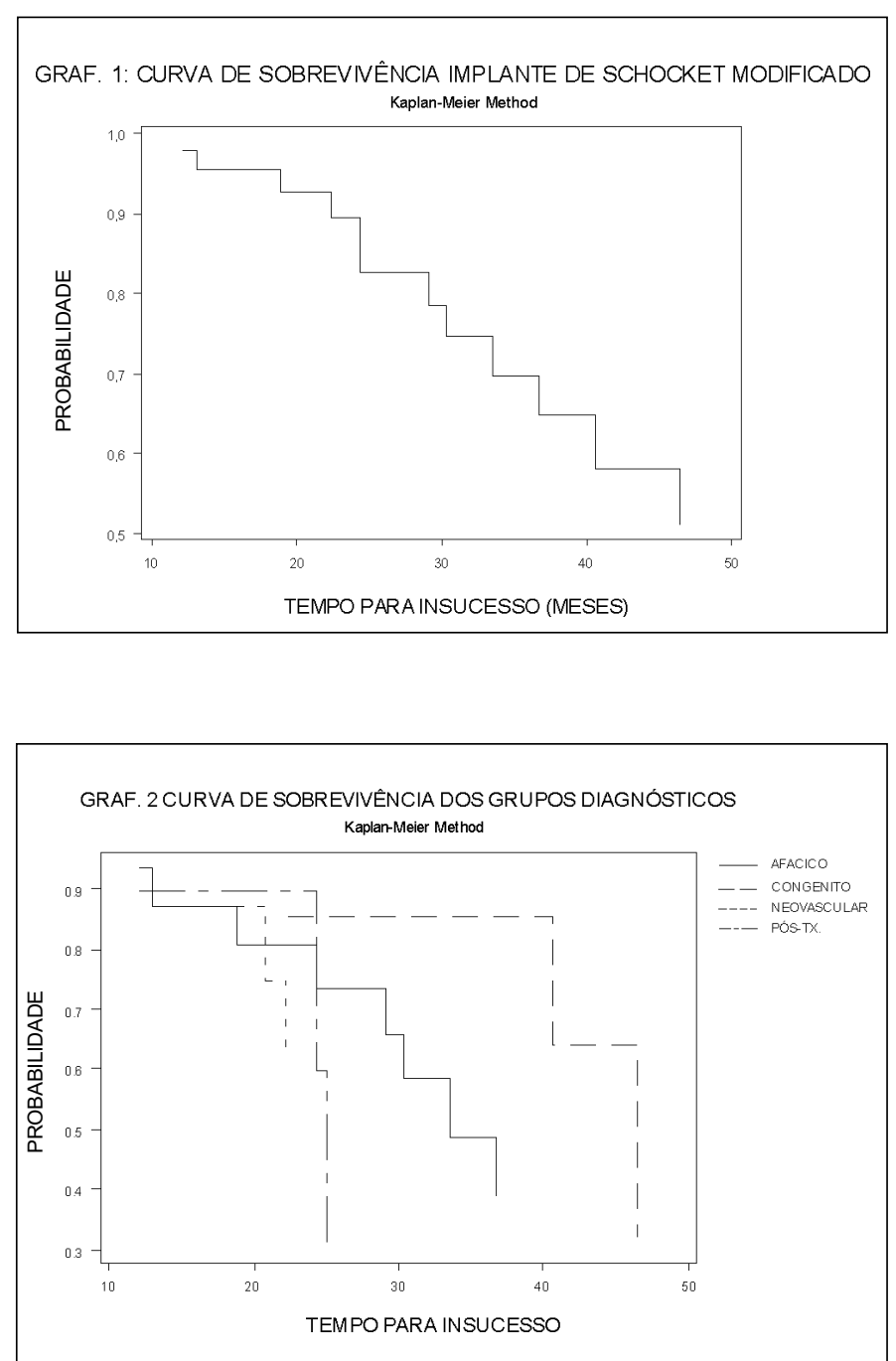

estatística $(p=0,004)$. Observou-se que a Po dos casos de glaucoma neovascular foi estatisticamente superior à dos demais. Para a Po final, a mesma análise revelou que as diferenças foram estatisticamente significantes $(p=0,006)$, sendo que os casos de glaucoma neovascular apresentaram Po inferior que os de glaucoma congênito e associado à afacia. A comparação da redução percentual da Po em relação à inicial

\begin{tabular}{|c|c|c|c|c|c|}
\hline \multicolumn{6}{|c|}{ Tabela III - Freqüência de sucesso por diagnóstico } \\
\hline & Afácico & Congênito & Neov. & Pós cerato. & $\mathbf{p}^{*}$ \\
\hline $\mathrm{n}$ & 16 & 7 & 10 & 10 & \\
\hline Sucesso & $8(50 \%)$ & $4(57,2 \%)$ & $7(70 \%)$ & $7(70 \%)$ & $0,6 \#$ \\
\hline Insucesso & 8 & $3(42,8 \%)$ & $3(70 \%)$ & $3(30 \%)$ & \\
\hline Acomp. pós-op (meses) & $33,2 \pm 21,5$ & $36,6 \pm 11,4$ & $32,1 \pm 22,1$ & $21,8 \pm 12,8$ & $0,3^{*}$ \\
\hline Po pré-op $(\mathrm{mmHg})$ & $35,2 \pm 07,9$ & $32,6 \pm 04,4$ & $48,0 \pm 08,7$ & $33,0 \pm 15,4$ & $0,004^{*}$ \\
\hline Po final $(\mathrm{mmHg})$ & $20,6 \pm 07,7$ & $20,0 \pm 11,2$ & $09,0 \pm 6,3$ & $15,1 \pm 07,8$ & $0,006^{*}$ \\
\hline Red \% & $36,1 \pm 26,8$ & $39,1 \pm 34,0$ & $81,9 \pm 11,5$ & $43,9 \pm 38,1$ & $0,002^{*}$ \\
\hline Tempo sobrevida & 30,2 meses & 41,7 meses & 21,3 meses & 23,5 meses & $0,8^{\dagger}$ \\
\hline
\end{tabular}


entre os grupos diagnósticos pela análise de variância mostrou diferenças estatisticamente significantes $(p=0,002)$, sendo que a maior redução percentual ocorreu em glaucomas neovasculares.

As complicações pós-operatórias observadas encontramse expostas na tabela IV, sendo a ocorrência principal o toque endotelial da porção intra-ocular do tubo $(20 \%)$.

\section{DISCUSSÃO}

Nesta série, o implante de Schocket modificado proporcionou o controle da Po em 62,2\% após um acompanhamento pós-operatório médio de quase três anos. Observou-se uma probabilidade de sucesso de 0,5 após praticamente quatro anos de pós-operatório, período este quase coincidente com o tempo de sobrevida médio (38,9 meses) obtidos pela análise de Kaplan-Meier. Julgando-se os vários fatores de risco para o insucesso cirúrgico, estas taxas podem ser consideradas bastante satisfatórias e inclusive, mostram-se mais favoráveis do que as descritas em outras séries na literatura com acompanhamento pós-operatório equivalente com o uso do implante de Molteno ${ }^{1,6}$.

Entretanto, a taxa de sucesso descrita neste estudo contrasta com a referida em estudo anteriormente publicado por Omi et $\mathrm{al}^{10}$ que analisaram 55 casos tratados com o implante de Schocket modificado. Naquele estudo observouse que 90,9\% dos casos apresentaram Po igual ou inferior a $21 \mathrm{mmHg}$ após um acompanhamento médio de 10,3 $\pm 5,4$ meses. Esta diferença pode ser explicada pelo fato de que nesta série os casos foram acompanhados por um período significativamente maior, o que influencia diretamente no sucesso do procedimento como tem sido descrito na literatura ${ }^{1-3}$.

Nas diversas modalidades de glaucoma em que o implante de Schocket modificado foi empregado, a comparação dos resultados descritos anteriormente por Omi et al. ${ }^{10}$ com os do presente estudo evidenciam algumas diferenças. Principalmente para os casos de glaucomas associado à afacia e congênito observou-se uma deteriorização significativa do resultado cirúrgico. Por outro lado, as taxas de sucesso descritas nesta série em glaucoma neovascular são mais satisfatórias. Provavelmente isto deveu-se a que nesta série somente foram incluídos casos com acompanhamento pósoperatório igual ou superior a um ano, tendo em vista cumprir o objetivo de analisar os resultados da técnica a longo prazo. Como casos de glaucoma neovascular geralmente mostram-se bastante refratários, o critério de seleção pode ter excluído vários casos em que o insucesso ocorreu precocemente.

Os resultados do presente estudo indicam que o implante de Schocket modificado propicia o controle de glaucomas refratários a longo prazo com taxas de sucesso satisfatórias.

\section{SUMMARY}

Purpose: To analyze long-term results of modified Schocket implant.

Methods: The records of 45 patients (45 surgeries) with at least one year follow-up who received a modified Schocket implant were analyzed. Comparisons were made regarding success rates (final IOP $22 \mathrm{mmHg}$ ), survival time, diagnosis, initial and final IOP, percentual postoperative intraocular pressure (IOP) reduction and complications.

Results: After a mean follow-up of $31.9 \pm 19.9$ months 28 cases (62.2\%) were considered successful. Mean survival time was 38.9 months and success probability around 30 months 0.74. Initial IOP was $37.2 \pm 11.3 \mathrm{mmHg}$ and final IOP was $16.3 \pm 9.3 \mathrm{mmHg}$ and postoperative IOP reduction was $49.6 \pm 32.6 \%$. Survival analysis in the different diagnosis groups (neovascular, congenital, aphakic, postkeratoplasty) did not reveal statistical significance. Conclusion: The results suggest that the modified Schocket

\begin{tabular}{|c|c|c|c|c|c|}
\hline \multicolumn{6}{|c|}{ Tabela IV - Complicações pós-operatórias } \\
\hline Complicação & Total & Afácico & Cong. & Neov. & Pós-cerat \\
\hline $\mathrm{n}$ & 45 & 16 & 7 & 10 & 10 \\
\hline Toque endotelial & $9(20 \%)$ & $4(25 \%)$ & $2(20 \%)$ & $1(10 \%)$ & $2(20 \%)$ \\
\hline Extrusão do impl. & $4(8,9 \%)$ & $1(6,2 \%)$ & $1(14,3 \%)$ & $1(10 \%)$ & - \\
\hline Phthisis & $3(6,7 \%)$ & - & - & $3(30 \%)$ & - \\
\hline Hemor. vítrea & $3(6,7 \%)$ & - & - & $3(30 \%)$ & - \\
\hline Hifema & $3(6,7 \%)$ & - & - & $3(30 \%)$ & - \\
\hline Exposiçao do tubo & $2(4,4 \%)$ & $1(6,2 \%)$ & - & - & $1(10 \%)$ \\
\hline Obstrução por vítreo & $2(4,4 \%)$ & $2(12,5 \%)$ & - & - & - \\
\hline Hipotonia & $2(4,4 \%)$ & $1(6,2 \%)$ & $1(14,3 \%)$ & - & - \\
\hline Rejeição Tx & $2(4,4 \%)$ & - & - & - & $2(20 \%)$ \\
\hline Desc. coróide & $2(4,4 \%)$ & $1(6,2 \%)$ & - & - & $1(10 \%)$ \\
\hline Desc. retina & $2(4,4 \%)$ & - & - & - & $2(20 \%)$ \\
\hline Catarata & $2(4,4 \%)$ & - & - & $2(20 \%)$ & - \\
\hline Seidel + & $2(4,4 \%)$ & - & $1(14,3 \%)$ & $1(10 \%)$ & - \\
\hline
\end{tabular}


implant provides satisfactory long-term control of refractory glaucomas.

Keywords: Glaucoma; Drainage devices.

\section{REFERÊNCIAS BIBLIOGRÁFICAS}

1. Lloyd MA, Sedlak T, Heuer DK, Minckler DS, Baerveldt G, Lee MB, Martone JF. Clinical experience with the single-plate Molteno implant in complicated glaucomas. Ophthalmology 1992;99:679-87.

2. Sidoti PA, Baerveldt G. Glaucoma drainage implants. Cur Op Ophthalmol 1994;5:59-98.

3. Melamed S, Fiore PM. Molteno implant surgery in refractory glaucoma. Surv Ophthalmol 1990;34:441-8.

4. Cohen R, Ameida GV, Omi CA, Mandia CJr, Kwitko S. Glaucoma e aniridia: experiência inicial. Quatro olhos tratados cirurgicamente com o im- plante de Schocket modificado. Arq Bras Oftal 1990;53:141-4.

5. Almeida GV, Omi CA, Mandia CJr, Cohen R, Kwitko S. Experiência inicial com o implante de Molteno em glaucomas refratários. Arq Bras Oftal 1990;53:101-4.

6. Almeida GV, Omi CA, Mandia CJr, Cohen R, Kwitko S. Resultados preliminares obtidos com o implante de Schocket modificado em 12 olhos portadores de glaucoma neovascular. Arq Bras Oftal 1990;53:189-92.

7. Prata JAJr, Mermoud A, LaBree L, Minckler DS. In-vitro and In-vivo Flow Characteristics of Glaucoma Drainage Implants. Ophthalmology, 1995;102: 894-904

8. Prata JAJr, Santos RCR, Minckler DS. Surface Area of Glaucoma Drainage Implants and Perfusion Flow Rates in Rabbit Eyes. J Glaucoma 1995;4:274-80.

9. Omi CA, Almeida GV, Kwitiko S, Cohen R, Mandia CJr. Implante de Schocket modificado para o controle do glaucoma pós-ceratoplastia penetrante. Arq Bras Oftal 1990;53:155-61.

10. Omi CA, Almeida GV, Kwitiko S, Cohen R, Mandia CJr . Modified Schocket implant for refractory glaucoma. Experience of 55 cases. Ophthalmology 1991;98:211-4.

\title{
I CONGRESSO PAN-AMERICANO DE TRAUMA OCULAR
}

\author{
22 a 24 de Março de 2001 \\ GRANDVILLE HOTEL \\ Belo Horizonte-MG
}

Promoção: Sociedade Pan-Americana de Trauma Ocular

Presidente: Dr. Christiano Barsante

Informações: Consult Comunicação e Marketing

Tel./Fax: (31) 3274-1550

e-mail: comunica@consultcom.com.br

internet: www.consultcom.com.br 